\title{
Effect of probiotic supplementation on productive performance of quail chicks.
}

\author{
GADO,S.M. ${ }^{1}$, EL-GENDI,G.M. ${ }^{1}$, MOHAMADY, T.F. ${ }^{2}$, EL-GARHY, O.H ${ }^{1}$ and EL-KHALEA, M.E \\ 1-Department of Animal Production, Faculty of Agriculture, Benha University \\ 2-Head of Biotechnology Lab, the Regional Center for Food and Feed, Agriculture Research center.
}

\begin{abstract}
Six hundred unsexed one day old Japanese quail chicks of a nearly similar live body weight were used in the present study as a trial to improve the productivity of quail chicks applying three probiotic preparations including (pediococcus acidilactici, pediococcus acidilactici plus entrococcus faecium and Bacillus licheniforis plus Bacillus Subtilis) to quail chicks, diet at a level of 0.0, 1.0, 1.5 and $2.0 \mathrm{~kg}$ probiotic/ton ration.

The results obtained showed that chicks fed diet supplemented with Bacillus licheniforis plus Bacillus Subtilis had the highest averages of body weight and weight gain compared with different treatment applied. Chicks received $1.5 \mathrm{~kg}$ probiotics /ton ration showed the highest averages of body weight and body weight gain at the end of the experimental period followed by chicks received $2 \mathrm{~kg} / \mathrm{ton}$, then by those fed diet with $1 \mathrm{~kg} / \mathrm{ton}$ when compared with the control group. Chicks fed diet supplemented with a mixture of Bacillus licheniforis and Bacillus Subtilis showed the lowest average of feed consumption at all estimated periods compared with those fed pediococcus alone or pediococcus plus entrococcus. The lowest average of feed consumption was observed when Bacillus licheniforis plus Bacillus Subtilis was interacted with either 1.5 or $2.0 \mathrm{~kg} / \mathrm{ton}$ ration, respectively. Supplementing diet with Bacillus licheniforis plus Bacillus Subtilis had significantly the better efficiency of feed utilization during the whole experimental period followed by those fed diet with pediococcus plus entrococcus and pediococcus alone, respectively. Chicks fed diet supplemented with Bacillus licheniforis plus Bacillus Subtilis had the better performance index and economical efficiency followed by those fed diet with pediococcus plus entrococcus and then by those fed pediococcus alon. In general all levels of feed additives applied had higher performance index and economical efficiency values compared to controls. The higher economical efficiency and performance index values were observed when Bacillus licheniforis plus Bacillus Subtilis was interacted with either 1.5 or $2 \mathrm{~kg} / \mathrm{ton}$ ration. The lowest average of mortality rate was observed in chicks received $1.5 \mathrm{~kg}$ probiotics per ton diet (3.88\%) followed by those fed $2 \mathrm{~kg} / \mathrm{ton}(4.99 \%)$, then by $1 \mathrm{~kg} / \mathrm{ton}$ (5.55\%)compared to control group(13.33\%). Bacillus licheniforis plus Bacillus Subtilis at a level of 1.5 and 2.0 $\mathrm{kg} \mathrm{ton} /$ ration seemed to be adequate to achieve the favorable results and could be recommended from the economic point of view.
\end{abstract}

Key words: "Quail, Probiotic, Body weight, Feed consumption, Feed conversion, Economic efficiency and Mortality"

\section{Introduction}

Probiotic microorganisms are inherently present in fermented food products and according to Kamiya et al., (2008) they are live microbial feed supplement which beneficially affect the host animals by improving their microbial balance, (Fuller, 1989) Probiotics is a live microbial feed supplement which beneficially affects the host animal by improving its intestinal microbial balance, helping newly-hatched chicks colonize normal microflora. The species being currently used in probiotic preparations are varied and many. A probiotic preparation may contain one or several different strains of microorganisms. The microorganisms used in animal feed are mainly gram postive bacterial strains belonging to the species Lactobacillus, Enterococcus, Pediococcus and Bacillus.

Some other probiotics are microscopic fungi such as strains of yeast belonging to the Saccharomyces cerevisiae species. Direct feed microbial product benefit the host animal by stimulating appetite (Nahashon et al., 1992 and Nahashon et al., 1993), improving intestinal microbial (Fuller, 1989) synthesis vitamins (Coates and Fuller., 1977), stimulate the immune system (Saarela et al., 2000), and stimulate lactic acid (Bailey, 1987). So the Probiotics is become a field of science, medicine, growth promoters and business that is growing rapidly.Addition of either pure lactobacillus cultures or mixtures of lactobacilli and other bacteria to poultry diets has produced variable results. The present trial was aimed to study the effect of dietary supplementation of probiotics on productive traits of Japanes quail.

\section{Materials and methods}

The present study was carried out at the Poultry Research Farm, Department of Animal Production, Faculty of Agriculture, Benha University. A total number of 600 unsexed one day old Japanese quail chicks of nearly similar live body weights were used in this study. Birds were weighed at hatch and leg banded. All chicks were kept under similar, standard hygienic and environmental condition. Brooding cages with gas heaters were used for brooding chicks. Brooding 
temperature was maintained at $37^{\circ} \mathrm{c}$ during the first five days of chick' $\mathrm{s}$ age then decreased by $2^{\circ} \mathrm{c}$ weekly until the end of brooding period. Chicks were vaccinated against Newcastle disease virus. No drugs or antibiotics were used in these experiments. Feed and water were offered ad-libitum. The basal diet (Table 1) was formulated according to the recommended requirements of NRC, (1994). Chicks were randomly divided into three groups each of 180 chicks each. Birds of the first, second and third groups were supplemented with Pediococcs acidilactici; Pediococcs acidilactici plus Enterococcus faecium (M74) and Bacillus licheniforis plus Bacillus Subtilis, respectively. Each group was then subdivided into three subgroups of 60 chicks each. They were treated with the previously mentioned probiotics at dose of $1.0,1.5$ and $2.0 \mathrm{~kg}$ probiotic per ton ration, respectively. Birds of the $10^{\text {th }}$ group was considered as control and fed basal diet without any supplementation.

Live body weight and feed consumption were weekly recorded and body weight gain and feed conversion were then calculated .Performance index was calculated according to North (1981).

Performance index $(\%)=$

Live body weight $(\mathrm{kg})$

Feed conversion X 100
efficiency $\quad$ (EE) was calculated according to the following equation:

$\mathrm{EE}=\{$ (price of $\mathrm{kg}$ weight gain $)-($ feed cost $/ \mathrm{kg}$ gain $) /$ feed cost/ kg gain) $\}$.

Percentage of mortality was calculated by subtreating the number of live birds at the end of experiment from the inithial total number according to the following formula:

Mortality rate $(\%)=\{(\mathrm{I}-\mathrm{E}) / \mathrm{I}\} \times 100$

Whereas:

I=Initial number of birds

$E=$ Number of live birds at the end of the experimental period

Data were statistically analyzed using general linear models procedures of SAS (1996).

The statistical model used was:

Xijk $=\boldsymbol{\mu}+\mathbf{T i}+\mathbf{L} \mathbf{j}+\mathbf{T L i j}+\mathbf{e i j k}$

Where;

$\boldsymbol{\mu}=$ overall mean

$\mathbf{T i}=$ the effect of the ith treatment applied

$\mathbf{L} \mathbf{j}=$ the effect of $\mathbf{j t h}$ level of probiotics

TLij = the effect of the interaction between the ith treatment and the jth levels of probiotics eijk $=$ the effect of random residual effect.

Table 1. Composition and calculated analysis of basal diet.

\begin{tabular}{lc}
\hline \multicolumn{1}{c}{ Ingredients } & $\boldsymbol{\%}$ \\
\hline Yellow corn & 56.00 \\
Soybean meal $(44 \%)$ & 27.90 \\
Corn gluten $(60 \%)$ & 12.00 \\
di-calcium phosphate & 1.70 \\
\hline
\end{tabular}

\begin{tabular}{lc}
\hline Calcium carbonate & 1.35 \\
Common salt & 0.25 \\
Hy-mix & 0.30 \\
premix* & \\
DL.methionine & \\
Lysine & 0.20 \\
Calculated analysis & 0.30 \\
ME $(\mathrm{kcal} / \mathrm{kg})$ & \\
$\mathrm{CP}(\%)$ & 2948.3 \\
calcium & 24.39 \\
Available phosphorus & 0.97 \\
$(\%)$ & 0.45
\end{tabular}

*Each $2.5 \mathrm{~kg}$ of vitamins and minerals mixture contains:12000.000 IU vitamin A acetate; 2000.000 IU vitamin D3;10.000 $\mathrm{mg}$ vitamin E acetate; $2000 \mathrm{mg}$ vitamin $\mathrm{K} 3 ; 100 \mathrm{mg}$ vitamin $\mathrm{B} 1 ; 4000 \mathrm{mg}$ vitamin B2; $1500 \mathrm{mg}$ vitamin B6; $10 \mathrm{mg}$ vitamin B12; $10.000 \mathrm{mg}$ pantothenic acid; $20.000 \mathrm{mg}$ Nicotinic acid; $1000 \mathrm{mg}$ Folic acid; $50 \mathrm{mg}$ Biotin; $500.000 \mathrm{mg}$ chorine; $10.000 \mathrm{mg}$ Copper; $1000 \mathrm{mg}$ Iodine; $300.00 \mathrm{mg}$ Iron; $55.000 \mathrm{mg}$ Manganese; $55.000 \mathrm{mg}$ Zinc ,and $100 \mathrm{mg}$ Selenium.

\section{Results and discussion}

\section{Body weight}

Data concerning the body weight of experimental chicks along the whole period of the study are presented in Table 2. Obtained data showed significant variation in average body weight due to treatments applied at the 2nd $(\mathrm{p}<0.05)$ and 6th $(p<0.001)$ weeks of age. Feeding birds diet supplemented with pediococcus strains as well as those of control group recorded the lowest average of body weight at all periods of estimation. However, birds fed diet supplemented with Bacillus licheniforis plus Bacillus Subtilis had the highest average of body weight that mounted 50.09, 114.74 and 199.20 $\mathrm{g}$ at $2 \mathrm{nd}$, 4th and 6th weeks of age, respectively. Chicks treated with pediococcus plus entrococcus recorded intermediate average of body weight estimated at the corresponding intervals. This may lead to conclude that mixture of two probiotics preparation showed efficient effect on improving body weight performance. This may be due to the biological effect of the two microbial preparations towards enhancement of body weight average as birds grew older.

Average body weight increased as the levels of dietary probiotics supplementation increased up to the level of $1.5 \mathrm{~kg} /$ ton ration. Chicks received $1.5 \mathrm{~kg}$ probiotics /ton ration showed the highest average of body weight $(201.81 \mathrm{~g})$ at the end of the experimental period followed be chicks received $2 \mathrm{~kg} /$ ton $(201.18$ g) then by those fed diet with $1 \mathrm{~kg} / \mathrm{ton}(198.16 \mathrm{~g})$ when compared with the control group (183.79 g). This may lead to state that the level of supplementation added to quail diet must not exceed $1.5 \mathrm{~kg} / \mathrm{ton}$ ration. The interaction effect between treatments applied and the levels of dietary supplementation was found to be of highly significant effect $(\mathrm{p}<0.001)$ on average body weight 
at the end of the experimental period only. The highest average of body weight was observed when Bacillus licheniforis plus Bacillus Subtilis interacted with 1.5 and $2.0 \mathrm{~kg} / \mathrm{ton}$ ration, respectively. The benefit effect of probiotics on live body weight may be attributed to the improvement that may occur in nutrients absorption and suppression of harmful bacteria, maintenance of normal intestinal microflora, increase in digestive enzyme activity, ammonia depletion, inhibition of growth of the entero-pathpgens in the gut by decreasing the intestinal ph and efficient utilization of nutrients by the beneficial microbes. (Fuller, 1989 and Jin et al.,
2000).Results obtained go in harmony with the finding of Mountzouris et al., (2007) who reported similar growth-promoting effects among birds fed avilamycin and birds administered a multi-species probiotic product (containing Lactobacillus reuteri, Enterococcus faecium, Bifidobacterium animalis, Pediococcus acidilactici, Lactobacillus salivarius) in feed and water. Also Abaza et al., (2008) reported that, applying either black seed oil, saccharomyce cervisiae or Bacillus licheniforis and Bacillus Subtilis to broiler diet increased body weight at 12 weeks of age as compared with controls.

Table 2. Least squares means and standard error (LSM \pm S.E) for body weight $(\mathrm{g})$ of birds of different experimental groups as affected by dietary supplements applied.

\begin{tabular}{|c|c|c|c|c|c|}
\hline \multirow{2}{*}{\multicolumn{2}{|c|}{$\begin{array}{l}\text { Independent variables } \\
\text { and interaction }\end{array}$}} & \multicolumn{4}{|c|}{ Body weight (g) at } \\
\hline & & Hatch & $2^{\text {nd }}$ week & $4^{\text {th }}$ week & $6^{\text {th }}$ week \\
\hline \multirow{3}{*}{$\begin{array}{c}\text { treatments } \\
(\mathrm{T})\end{array}$} & (T1) & $7.47 \pm 0.29$ & $49.67 \pm 0.29^{b}$ & $114.07 \pm 0.29$ & $192.39 \pm 0.29^{\mathrm{c}}$ \\
\hline & (T2) & $7.45 \pm 0.29$ & $50.04 \pm 0.29^{b}$ & $114.35 \pm 0.29$ & $197.11 \pm 0.29^{b}$ \\
\hline & (T3) & $7.44 \pm 0.29$ & $50.09 \pm 0.29^{a}$ & $114.74 \pm 0.29$ & $199.20 \pm 0.29^{\mathrm{a}}$ \\
\hline \multirow{4}{*}{$\begin{array}{c}\text { levels } \\
(\mathrm{L})\end{array}$} & LO( Control) & $7.44 \pm 0.33$ & $48.19 \pm 0.33^{\mathrm{c}}$ & $111.29 \pm 0.33^{b}$ & $183.79 \pm 0.33^{\mathrm{c}}$ \\
\hline & $\mathrm{L} 1(1.0 \mathrm{~kg} /$ ton $)$ & $7.45 \pm 0.33$ & $50.02 \pm 0.33^{b}$ & $115.08 \pm 0.33^{\mathrm{a}}$ & $198.16 \pm 0.33^{b}$ \\
\hline & $\mathrm{L} 2(1.5 \mathrm{~kg} / \mathrm{ton})$ & $7.49 \pm 0.33$ & $51.64 \pm 0.33^{\mathrm{a}}$ & $111.80 \pm 0.33^{\mathrm{a}}$ & $201.81 \pm 0.33^{\mathrm{a}}$ \\
\hline & $\mathrm{L} 3(2.0 \mathrm{~kg} / \mathrm{ton})$ & $7.43 \pm 0.33$ & $51.01 \pm 0.33^{\mathrm{a}}$ & $115.37 \pm 0.33^{\mathrm{a}}$ & $201.18 \pm 0.33^{\mathrm{a}}$ \\
\hline \multirow{12}{*}{$\begin{array}{c}\text { Interaction } \\
(\mathbf{T} \times \mathbf{L})\end{array}$} & $\mathrm{T} 1 \times \mathrm{L} 0$ & $7.44 \pm 0.33$ & $48.19 \pm 0.33$ & $111.29 \pm 0.33$ & $183.79 \pm 0.33^{\mathrm{c}}$ \\
\hline & $\mathrm{T} 1 \times \mathrm{L} 1$ & $7.47 \pm 0.58$ & $49.57 \pm 0.58$ & $114.66 \pm 0.58$ & $195.78 \pm 0.58^{\mathrm{e}}$ \\
\hline & $\mathrm{T} 1 \times \mathrm{L} 2$ & $7.52 \pm 0.58$ & $50.78 \pm 0.58$ & $115.26 \pm 0.58$ & $195.60 \pm 0.58^{\mathrm{e}}$ \\
\hline & $\mathrm{T} 1 \times \mathrm{L} 3$ & $7.45 \pm 0.58$ & $50.52 \pm 0.58$ & $115.59 \pm 0.58$ & $194.40 \pm 0.58^{\mathrm{e}}$ \\
\hline & $\mathrm{T} 2 \times \mathrm{L} 0$ & $7.44 \pm 0.33$ & $48.19 \pm 0.33$ & $111.29 \pm 0.33$ & $183.79 \pm 0.33^{\mathrm{c}}$ \\
\hline & $\mathrm{T} 2 \times \mathrm{L} 1$ & $7.42 \pm 0.58$ & $49.22 \pm 0.58$ & $115.17 \pm 0.58$ & $198.43 \pm 0.58^{d}$ \\
\hline & $\mathrm{T} 2 \times \mathrm{L} 2$ & $7.50 \pm 0.58$ & $51.72 \pm 0.58$ & $115.86 \pm 0.58$ & $202.93 \pm 0.58^{b}$ \\
\hline & T2 $\times$ L3 & $7.43 \pm 0.58$ & $51.03 \pm 0.58$ & $115.09 \pm 0.58$ & $203.28 \pm 0.58^{b}$ \\
\hline & $\mathrm{T} 3 \times \mathrm{L} 0$ & $7.44 \pm 0.33$ & $48.19 \pm 0.33$ & $111.29 \pm 0.33$ & $183.79 \pm 0.33^{\mathrm{c}}$ \\
\hline & $\mathrm{T} 3 \times \mathrm{L} 1$ & $7.44 \pm 0.58$ & $51.29 \pm 0.58$ & $115.43 \pm 0.58$ & $200.26 \pm 0.58^{a}$ \\
\hline & T3× L2 & $7.46 \pm 0.58$ & $52.41 \pm 0.58$ & $116.29 \pm 0.58$ & $206.90 \pm 0.58^{a}$ \\
\hline & T3× L3 & $7.42 \pm 0.58$ & $51.72 \pm 0.58$ & $115.95 \pm 0.58$ & $205.86 \pm 0.58^{c}$ \\
\hline
\end{tabular}

(A ,b ,c ,... ) Means within the same letter in each column are not significantly different.

(T1)Pediococcus

(T2) pediococcus plus entrococcus

(T3) Bacillus licheniforis plus Bacillus Subtilis

\section{Body weight gain}

Data obtained (Table 3) revealed highly significant variation in average body weight gain due to treatments applied. It was quite true at all intervals of estimation except at the period from (2-4) weeks of age only. On the other hand, the higher body weight gain was observed during the whole period (0-6) in the group of chicks fed Bacillus licheniforis plus Bacillus Subtilis (191.57g) when compared with those fed pediococcus plus entrococcus $(189.77 \mathrm{~g}$ ) or pediococcus (184.93g) (Table 3). However, no significant variation was found in average body weight gain in chicks fed pediococcus plus entrococcus and those fed Bacillus licheniforis plus Bacillus Subtilis during the same period. Generally, applying probiotics at different levels increased average body weight gain during all periods of estimation as well as during the whole experimental period compared to the controls. Quail chicks fed diet with $1.5 \mathrm{~kg} / \mathrm{ton}$ ration showed the highest average of body weight gain ( $194.18 \mathrm{~g}$ ) followed by those fed $2 \mathrm{~kg} /$ ton ( 193.84g) and $1 \mathrm{~kg} / \mathrm{ton}$ (190.71g). On the other hand, control group showed the lowest average of body weight gain (176.35g). Average body weight gain increased as the level of dietary supplements increased up to the level of $1.5 \mathrm{~kg} / \mathrm{ton}$ ration (Table 3) then decreased with insignificant magnitude when supplementation was increased up to $2.0 \mathrm{~kg} /$ ton ration. This mean that the biological effect of dietary supplementation applied reach its peak up to the level of $1.5 \mathrm{~kg} /$ ton ration then the rate decreased as the level of dietary probiotic increased. These results are in agreement with those obtained by Jin et al., (1996) who reported that, using 
Bacillus Subtilis as probiotics in the experimental diet increased weight gain as compared to untreated birds. The same results were reported by Asmita et al., (2001); Tarun,(2008) ; Chimote et al.,(2009) and Al-homidan et al., (2010) who found that, supplementation of probiotic(Bacillus sp, Lactobacillus and Pediococcus) and enzymes significantly $(\mathrm{P}<0.01)$ improved live body weights and weight gain as compared to control quails . In contrast, Namra et al., (2005) and Willis et al., (2007), reported that using these additives in the broiler ration had no significant effects on growth performance.

\section{Feed consumption}

Treatments applied were found to have highly significant effect $(p<0.001)$ on average of feed consumption during all experimental periods except at the period from (4-6) weeks of age (Table 4).Chicks fed diet supplemented with a mixture of Bacillus licheniforis plus Bacillus Subtilis showed the lowest average of feed consumption at all estimated periods compared with those fed Pediococcus alone or Pediococcus plus entrococcus (Table 4). Average of feed consumption decreased as the level of feed supplements increased (Table 4). Birds fed diet supplemented with probiotics at a level of $2 \mathrm{~kg}$ per ton ration showed the lowest average of feed consumption when compared with controls or those of the other two levels applied (Table, 4). Significant variation was also observed due to the interaction effect between treatments and the level of feed supplementation applied at all experimental intervals. The lowest average of feed consumption was observed when Bacillus licheniforis plus Bacillus Subtilis was interacted with either 1.5 or 2.0 $\mathrm{kg} /$ ton ration, respectively. Results obtained agree with those reported by Santoso et al., (2005) and Hideya and Taku, (2004) who cleared that diet supplemented with either Bacillus Subtilis or Bacillus cereus toyoi decreased feed intake. The same findings were obtained by Asmita et al. (2001) and Awad et al., (2009) who stated that feed consumption decreased as result of supplementing birds feed with probiotics preparation. The reduction in feed consumption and improvement in growth performance in treated quail chicks may be attributed to enhancement in the metabolic efficiency and feed utilization rather than to the amount of feed consumed by the chicks.

\section{Feed conversion}

Obtained data tabulated in (Table 5) showed that highly significant variation in feed conversion was detected due to the various dietary probiotics applied. Supplementing diet with Bacillus licheniforis plus Bacillus Subtilis had the better efficiency on feed utilization during the period from $(0-2)$ weeks
(1.74),(2-4) weeks (3.03) and( 4-6 ) weeks (3.62 g feed/g gain) as well as during the whole experimental period: (0-6)weeks (2.99 $\mathrm{g}$ feed/g gain) followed by those fed diet with pediococcus plus entrococcus 1.86, 3.05,3.69 and 3.06 $\mathrm{g}$ feed /g gain, respectively and when fed pediococcus alon 1.89, 3.09, 3.87 and $3.14 \mathrm{~g}$ feed /g gain, respectively( Table 5 ).

It may be concluded that the biological action of the feed supplements applied lowered the amount of feed consumed and increased the average body weight gain with the same rate in all treatments applied. In addition, all dietary supplements act on the metabolic pathway by increasing the enzymatic activity that may improve the feed utilization Yanbo and Qing (2010) found significant improvement in feed conversion $(\mathrm{P}<0.05)$ due to probiotics applied in birds diet during the experimental period. They attributed this improvement to the higher digestive enzyme activity (protease, amylase and lipase) that may occur. Feed conversion significantly improved as the level of dietary supplementation increased. Chick's received $2 \mathrm{~kg} / \mathrm{ton}$ ration recorded the better feed efficiency (2.93 g feed /g gain) compared to those received $1.5 \mathrm{~kg} / \mathrm{ton}$ ration $(2.95 \mathrm{~g}$ feed $/ \mathrm{g}$ gain), $1 \mathrm{~kg} / \mathrm{ton}$ (3.04) and controls (3.34 $\mathrm{g}$ feed/g gain) during the whole period of estimation (Table 5). This may lead to recommend applying this level if good feed utilization was aimed. On the other hand, highly significant effect $(\mathrm{P}<0.001)$ was found due to the interaction between treatments and levels of feed supplementation all over the experimental period. However, this interaction effect differed according to treatments applied. Applying Bacillus licheniforis plus Bacillus Subtilis at a level of 1.5 or $2.0 \mathrm{~kg} / \mathrm{ton}$ ration, respectively followed by pediococcus plus entrococcus when applied with a level of $2 \mathrm{~kg} / \mathrm{ton}$ ration were mostly the most favorable level that may be recommended. However, applying Bacillus licheniforis plus Bacillus Subtilis ration at a level of $1.5 \mathrm{~kg} / \mathrm{ton}$ was found to be better (Table 5). The positive responses to probiotic may be attributed to more ideal intestinal flora effect that improved feed conversion. Obtained results agree with those of Sanders and Veld (1999) who suggested that the use of multistrain and multispecies probiotics might be more effective than monostrain probiotics. Silva $e t$ al., (2000) ;Pelicano et al., (2005); Franco et al., (2005) and Chafai et al., (2007) demonstrated an improvement in feed conversion in chickens which fed on probiotics such as Bacillus subtilis, Lactobacillus acidophilus, Saccharomyces cerevisiae , P. acidilactici and Enterococcus faecium.. 
Table 3. Least squares means and standard error ( $\mathrm{LSM} \pm \mathrm{S}$.E) for body weight gain $(\mathrm{g})$ of birds of different experimental groups as affected by dietary supplements applied.

\begin{tabular}{|c|c|c|c|c|c|}
\hline \multirow{2}{*}{\multicolumn{2}{|c|}{$\begin{array}{l}\text { Independent variables } \\
\text { and interaction }\end{array}$}} & \multicolumn{4}{|c|}{ Body weight gain (g) at } \\
\hline & & (0-2) weeks & (2-4) weeks & (4-6) weeks & $(0-6)$ weeks \\
\hline \multirow{3}{*}{$\begin{array}{l}\text { treatments } \\
(\mathrm{T})\end{array}$} & (T1) & $42.30 \pm 0.28^{b}$ & $64.31 \pm 0.28$ & $78.32 \pm 0.28^{\mathrm{c}}$ & $184.93 \pm 0.28^{b}$ \\
\hline & (T2) & $42.59 \pm 0.28^{b}$ & $64.42 \pm 0.28$ & $82.75 \pm 0.28^{b}$ & $189.77 \pm 0.28^{\mathrm{a}}$ \\
\hline & (T3) & $43.46 \pm 0.28^{\mathrm{a}}$ & $63.65 \pm 0.28$ & $84.64 \pm 0.28^{\mathrm{a}}$ & $191.57 \pm 0.28^{\mathrm{a}}$ \\
\hline \multirow{4}{*}{$\begin{array}{l}\text { levels } \\
(\mathrm{L})\end{array}$} & LO( Control) & $40.75 \pm 0.33^{\mathrm{c}}$ & $63.10 \pm 0.33^{\mathrm{c}}$ & $72.50 \pm 0.33^{\mathrm{c}}$ & $176.35 \pm 0.33^{\mathrm{c}}$ \\
\hline & $\mathrm{L} 1(1.0 \mathrm{~kg} /$ ton $)$ & $42.58 \pm 0.33^{b}$ & $65.06 \pm 0.33^{\mathrm{a}}$ & $83.07 \pm 0.33^{b}$ & $190.71 \pm 0.33^{b}$ \\
\hline & $\mathrm{L} 2(1.5 \mathrm{~kg} / \mathrm{ton})$ & $44.21 \pm 0.33^{\mathrm{a}}$ & $63.92 \pm 0.33^{b c}$ & $86.00 \pm 0.33^{\mathrm{a}}$ & $194.18 \pm 0.33^{\mathrm{a}}$ \\
\hline & $\mathrm{L} 3(2.0 \mathrm{~kg} /$ ton $)$ & $43.60 \pm 0.33^{\mathrm{a}}$ & $64.43 \pm 0.33^{\text {ba }}$ & $85.80 \pm 0.33^{\mathrm{a}}$ & $193.84 \pm 0.33^{\mathrm{a}}$ \\
\hline \multirow{12}{*}{$\begin{array}{l}\text { Interaction } \\
(\mathbf{T} \times \mathbf{L})\end{array}$} & $\mathrm{T} 1 \times \mathrm{L} 0$ & $40.75 \pm 0.33$ & $63.10 \pm 0.33$ & $72.50 \pm 0.33^{f}$ & $176.35 \pm 0.33^{\mathrm{c}}$ \\
\hline & $\mathrm{T} 1 \times \mathrm{L} 1$ & $40.10 \pm 0.58$ & $65.09 \pm 0.58$ & $81.12 \pm 0.58^{\mathrm{d}}$ & $188.31 \pm 0.58^{\mathrm{cd}}$ \\
\hline & $\mathrm{T} 1 \times \mathrm{L} 2$ & $43.36 \pm 0.58$ & $64.48 \pm 0.58$ & $80.35 \pm 0.58^{\mathrm{de}}$ & $188.19 \pm 0.58^{\mathrm{cd}}$ \\
\hline & $\mathrm{T} 1 \times \mathrm{L} 3$ & $43.00 \pm 0.58$ & $64.57 \pm 0.58$ & $79.31 \pm 0.58^{\mathrm{e}}$ & $186.88 \pm 0.58^{\mathrm{d}}$ \\
\hline & $\mathrm{T} 2 \times \mathrm{LO}$ & $40.75 \pm 0.33$ & $63.10 \pm 0.33$ & $72.50 \pm 0.33^{f}$ & $176.35 \pm 0.33^{\mathrm{c}}$ \\
\hline & $\mathrm{T} 2 \times \mathrm{L} 1$ & $41.78 \pm 0.58$ & $65.95 \pm 0.58$ & $83.26 \pm 0.58^{\mathrm{c}}$ & $190.99 \pm 0.58^{\mathrm{bcd}}$ \\
\hline & $\mathrm{T} 2 \times \mathrm{L} 2$ & $44.30 \pm 0.58$ & $64.14 \pm 0.58$ & $87.07 \pm 0.58^{b}$ & $195.51 \pm 0.58^{\mathrm{ab}}$ \\
\hline & $\mathrm{T} 2 \times \mathrm{L} 3$ & $43.53 \pm 0.58$ & $64.52 \pm 0.58$ & $88.19 \pm 0.58^{b}$ & $196.24 \pm 0.58^{\mathrm{ab}}$ \\
\hline & $\mathrm{T} 3 \times \mathrm{L} 0$ & $40.75 \pm 0.33$ & $63.10 \pm 0.33$ & $72.50 \pm 0.33^{f}$ & $176.35 \pm 0.33^{\mathrm{c}}$ \\
\hline & $\mathrm{T} 3 \times \mathrm{L} 1$ & $43.86 \pm 0.58$ & $64.14 \pm 0.58$ & $84.83 \pm 0.58^{\mathrm{c}}$ & $192.83 \pm 0.58^{\mathrm{bc}}$ \\
\hline & $\mathrm{T} 3 \times \mathrm{L} 2$ & $44.97 \pm 0.58$ & $63.14 \pm 0.58$ & $90.60 \pm 0.58^{\mathrm{a}}$ & $198.71 \pm 0.58^{\mathrm{a}}$ \\
\hline & T3× L3 & $44.27 \pm 0.58$ & $64.22 \pm 0.58$ & $89.91 \pm 0.58^{\mathrm{a}}$ & $198.41 \pm 0.58^{\mathrm{a}}$ \\
\hline
\end{tabular}

$(\mathrm{A}, \mathrm{b}, \mathrm{c}, . .$.$) Means within the same letter in each column are not significantly different$

(T1)Pediococcus

(T2) pediococcus plus entrococcus

(T3) Bacillus licheniforis plus Bacillus Subtilis

Table 4. Least squares means and standard error $(\mathrm{LSM} \pm \mathrm{S} . \mathrm{E})$ for feed consumption $(\mathrm{g})$ of birds of different experimental groups as affected by dietary supplements applied.

\begin{tabular}{|c|c|c|c|c|c|}
\hline \multirow{2}{*}{\multicolumn{2}{|c|}{$\begin{array}{l}\text { Independent variables } \\
\text { and interaction }\end{array}$}} & \multicolumn{4}{|c|}{ Average feed consumption (g/bird/day) at } \\
\hline & & $(0-2)$ weeks & (2-4) weeks & (4-6) weeks & (0-6 )weeks \\
\hline \multirow{3}{*}{$\begin{array}{l}\text { treatments } \\
(\mathrm{T})\end{array}$} & (T1) & $5.72 \pm 0.04^{\mathrm{a}}$ & $14.22 \pm 0.04^{\mathrm{a}}$ & $21.59 \pm 0.04$ & $581.76 \pm 1.73^{\mathrm{a}}$ \\
\hline & (T2) & $5.66 \pm 0.04^{\mathrm{a}}$ & $14.05 \pm 0.04^{b}$ & $21.67 \pm 0.04$ & $579.46 \pm 1.73^{\mathrm{a}}$ \\
\hline & (T3) & $5.41 \pm 0.04^{\mathrm{b}}$ & $13.80 \pm 0.04^{\mathrm{c}}$ & $21.64 \pm 0.04$ & $572.08 \pm 1.73^{b}$ \\
\hline \multirow{4}{*}{$\begin{array}{c}\text { levels } \\
(\mathrm{L})\end{array}$} & LO( Control) & $5.71 \pm 0.04^{\mathrm{a}}$ & $14.32 \pm 0.04^{\mathrm{a}}$ & $22.05 \pm 0.04^{\mathrm{a}}$ & $589.20 \pm 2.00^{\mathrm{a}}$ \\
\hline & $\mathrm{L} 1(1.0 \mathrm{~kg} / \mathrm{ton})$ & $5.61 \pm 0.04^{\text {ab }}$ & $14.13 \pm 0.04^{\mathrm{b}}$ & $21.66 \pm 0.04^{b}$ & $579.77 \pm 2.00^{b}$ \\
\hline & L2 $(1.5 \mathrm{~kg} / \mathrm{ton})$ & $5.54 \pm 0.04^{b}$ & $13.91 \pm 0.04^{\mathrm{c}}$ & $21.47 \pm 0.04^{\mathrm{c}}$ & $573.12 \pm 2.00^{\mathrm{c}}$ \\
\hline & $\mathrm{L} 3(2.0 \mathrm{~kg} / \mathrm{ton})$ & $5.53 \pm 0.04^{b}$ & $13.74 \pm 0.04^{\mathrm{d}}$ & $21.36 \pm 0.04^{\mathrm{c}}$ & $568.97 \pm 2.00^{\mathrm{c}}$ \\
\hline \multirow{12}{*}{$\begin{array}{l}\text { Interaction } \\
(\mathbf{T} \times \mathbf{L})\end{array}$} & $\mathrm{T} 1 \times \mathrm{L} 0$ & $5.71 \pm 0.08^{\mathrm{ab}}$ & $14.32 \pm 0.08^{\mathrm{a}}$ & $22.05 \pm 0.08^{\mathrm{a}}$ & $589.20 \pm 3.46^{\mathrm{a}}$ \\
\hline & $\mathrm{T} 1 \times \mathrm{L} 1$ & $5.91 \pm 0.08^{\mathrm{a}}$ & $14.39 \pm 0.08^{\mathrm{a}}$ & $21.43 \pm 0.08^{\mathrm{cd}}$ & $584.32 \pm 3.46^{\mathrm{ab}}$ \\
\hline & $\mathrm{T} 1 \times \mathrm{L} 2$ & $5.7 \pm 10.08^{\mathrm{ab}}$ & $14.48 \pm 0.08^{\mathrm{a}}$ & $21.49 \pm 30.08^{\mathrm{cd}}$ & $582.72 \pm 3.46^{\mathrm{ab}}$ \\
\hline & $\mathrm{T} 1 \times \mathrm{L} 3$ & $5.57 \pm 0.08^{b c}$ & $13.71 \pm 0.08^{b}$ & $21.48 \pm 0.08^{\mathrm{c}}$ & $570.80 \pm 3.46^{\mathrm{cd}}$ \\
\hline & $\mathrm{T} 2 \times \mathrm{LO}$ & $5.71 \pm 0.08^{\mathrm{ab}}$ & $14.32 \pm 0.08^{\mathrm{a}}$ & $22.05 \pm 0.08^{\mathrm{a}}$ & $589.20 \pm 3.46^{\mathrm{a}}$ \\
\hline & $\mathrm{T} 2 \times \mathrm{L} 1$ & $5.57 \pm 0.08^{b c}$ & $14.28 \pm 0.08^{\mathrm{a}}$ & $21.57 \pm 0.08^{b c}$ & $580.00 \pm 3.46^{\mathrm{abc}}$ \\
\hline & $\mathrm{T} 2 \times \mathrm{L} 2$ & $5.65 \pm 0.08 \mathrm{a}^{\mathrm{b}}$ & $13.90 \pm 0.08^{b}$ & $21.74 \pm 0.08^{b}$ & $578.20 \pm 3.46^{\mathrm{abc}}$ \\
\hline & T2x L3 & $5.71 \pm 0.08 \mathrm{a}^{\mathrm{b}}$ & $13.71 \pm 0.08^{b}$ & $21.31 \pm 0.08^{\mathrm{cd}}$ & $570.44 \pm 3.46^{\mathrm{cd}}$ \\
\hline & $\mathrm{T} 3 \times \mathrm{LO}$ & $5.71 \pm 0.08^{\mathrm{ab}}$ & $14.32 \pm 0.08^{\mathrm{a}}$ & $22.05 \pm 0.08^{\mathrm{a}}$ & $589.20 \pm 3.46^{\mathrm{a}}$ \\
\hline & $\mathrm{T} 3 \times \mathrm{L} 1$ & $5.35 \pm 0.08^{\mathrm{cd}}$ & $13.71 \pm 0.08^{b}$ & $22.00 \pm 0.08^{\mathrm{a}}$ & $575.00 \pm 3.46^{\mathrm{bcc}}$ \\
\hline & $\mathrm{T} 3 \times \mathrm{L} 2$ & $5.25 \pm 0.08^{\mathrm{d}}$ & $13.37 \pm 0.08^{\mathrm{c}}$ & $21.26 \pm 0.08^{d}$ & $558.44 \pm 3.46 \mathrm{e}$ \\
\hline & T3× L3 & $5.31 \pm 0.08^{\mathrm{cd}}$ & $13.80 \pm 0.08^{\mathrm{d}}$ & $21.28 \pm 0.08^{\mathrm{d}}$ & $565.68 \pm 3.46^{\mathrm{ed}}$ \\
\hline
\end{tabular}

$(\mathrm{A}, \mathrm{b}, \mathrm{c}, \ldots$.$) Means within the same letter in each column are not significantly different$

(T1)Pediococcus

(T2) pediococcus plus entrococcus

(T3) Bacillus licheniforis plus Bacillus Subtilis 
Table 5. Least squares means and standard error (LSM \pm S.E) for feed conversion of birds of different experimental groups as affected by dietary supplements applied.

\begin{tabular}{|c|c|c|c|c|c|}
\hline \multirow{2}{*}{\multicolumn{2}{|c|}{$\begin{array}{l}\text { Independent variables } \\
\text { and interaction }\end{array}$}} & \multicolumn{4}{|c|}{ Average feed conversion ( $\mathrm{g}$ feed / g gain) at } \\
\hline & & $(0-2)$ weeks & (2-4) weeks & (4-6) weeks & (0-6) weeks \\
\hline \multirow{3}{*}{$\begin{array}{c}\text { treatments } \\
(\mathrm{T})\end{array}$} & (T1) & $1.89 \pm 0.01^{\mathrm{a}}$ & $3.09 \pm 0.01^{\mathrm{a}}$ & $3.87 \pm 0.01^{\mathrm{a}}$ & $3.14 \pm 0.01^{\mathrm{a}}$ \\
\hline & (T2) & $1.86 \pm 0.01^{\mathrm{b}}$ & $3.05 \pm 0.01^{\mathrm{b}}$ & $3.69 \pm 0.01^{b}$ & $3.06 \pm 0.01^{b}$ \\
\hline & (T3) & $1.74 \pm 0.01^{\mathrm{c}}$ & $3.03 \pm 0.01^{\mathrm{c}}$ & $3.62 \pm 0.01^{\mathrm{c}}$ & $2.99 \pm 0.01^{\mathrm{c}}$ \\
\hline \multirow{4}{*}{$\begin{array}{c}\text { levels } \\
(\mathrm{L})\end{array}$} & LO( Control) & $1.96 \pm 0.01^{\mathrm{a}}$ & $3.18 \pm 0.01^{\mathrm{a}}$ & $4.26 \pm 0.01^{\mathrm{a}}$ & $3.34 \pm 0.01^{\mathrm{a}}$ \\
\hline & $\mathrm{L} 1(1.0 \mathrm{~kg} / \mathrm{ton})$ & $1.84 \pm 0.01^{b}$ & $3.04 \pm 0.01^{b}$ & $3.65 \pm 0.01^{b}$ & $3.04 \pm 0.01^{b}$ \\
\hline & $\mathrm{L} 2(1.5 \mathrm{~kg} / \mathrm{ton})$ & $1.75 \pm 0.01^{\mathrm{c}}$ & $3.04 \pm 0.01^{\mathrm{b}}$ & $3.50 \pm 0.01^{\mathrm{c}}$ & $2.95 \pm 0.01^{\mathrm{c}}$ \\
\hline & $\mathrm{L} 3(2.0 \mathrm{~kg} / \mathrm{ton})$ & $1.77 \pm 0.01^{\mathrm{d}}$ & $2.98 \pm 0.01^{\mathrm{d}}$ & $3.49 \pm 0.01^{\mathrm{c}}$ & $2.93 \pm 0.01^{\mathrm{d}}$ \\
\hline \multirow{12}{*}{$\begin{array}{c}\text { Interaction } \\
(\text { TX L) }\end{array}$} & $\mathrm{T} 1 \times \mathrm{L} 0$ & $1.96 \pm 0.01^{\mathrm{a}}$ & $3.18 \pm 0.01^{\mathrm{a}}$ & $4.26 \pm 0.01^{\mathrm{a}}$ & $3.34 \pm 0.01^{\mathrm{a}}$ \\
\hline & $\mathrm{T} 1 \times \mathrm{L} 1$ & $1.97 \pm 0.01^{\mathrm{a}}$ & $3.09 \pm 0.01^{\mathrm{c}}$ & $3.70 \pm 0.01^{\mathrm{c}}$ & $3.10 \pm 0.01^{b}$ \\
\hline & $\mathrm{T} 1 \times \mathrm{L} 2$ & $1.84 \pm 0.01^{\mathrm{c}}$ & $3.14 \pm 0.01^{b}$ & $3.73 \pm 0.01^{\mathrm{c}}$ & $3.09 \pm 0.01^{b}$ \\
\hline & $\mathrm{T} 1 \times \mathrm{L} 3$ & $1.81 \pm 0.01^{\mathrm{d}}$ & $2.97 \pm 0.01^{\mathrm{f}}$ & $3.79 \pm 0.01^{b}$ & $3.05 \pm 0.01^{\mathrm{c}}$ \\
\hline & $\mathrm{T} 2 \times \mathrm{LO}$ & $1.96 \pm 0.01^{\mathrm{a}}$ & $3.18 \pm 0.01^{\mathrm{a}}$ & $4.26 \pm 0.01^{\mathrm{a}}$ & $3.34 \pm 0.01^{\mathrm{a}}$ \\
\hline & T2x L1 & $1.86 \pm 0.01^{b}$ & $3.03 \pm 0.01^{\mathrm{d}}$ & $3.63 \pm 0.01^{\mathrm{d}}$ & $3.03 \pm 0.01^{\mathrm{c}}$ \\
\hline & T2 $\times$ L2 & $1.78 \pm 0.01^{\mathrm{e}}$ & $3.03 \pm 0.01^{\mathrm{d}}$ & $3.49 \pm 0.01^{\mathrm{e}}$ & $2.95 \pm 0.01^{\mathrm{d}}$ \\
\hline & $\mathrm{T} 2 \times \mathrm{L} 3$ & $1.83 \pm 0.01^{\mathrm{c}}$ & $2.97 \pm 0.01^{\mathrm{f}}$ & $3.38 \pm 0.01^{f}$ & $2.90 \pm 0.01^{\mathrm{e}}$ \\
\hline & $\mathrm{T} 3 \times \mathrm{LO}$ & $1.96 \pm 0.01^{\mathrm{a}}$ & $3.18 \pm 0.01^{\mathrm{a}}$ & $4.26 \pm 0.01^{\mathrm{a}}$ & $3.34 \pm 0.01^{\mathrm{a}}$ \\
\hline & $\mathrm{T} 3 \times \mathrm{L} 1$ & $1.71 \pm 0.01^{\mathrm{f}}$ & $2.99 \pm 0.01^{\mathrm{ef}}$ & $3.63 \pm 0.01^{\mathrm{d}}$ & $2.98 \pm 0.01^{\mathrm{d}}$ \\
\hline & $\mathrm{T} 3 \times \mathrm{L} 2$ & $1.63 \pm 0.01^{\mathrm{h}}$ & $2.96 \pm 0.01^{\mathrm{f}}$ & $3.28 \pm 0.01^{\mathrm{g}}$ & $2.81 \pm 0.01^{\mathrm{g}}$ \\
\hline & T3× L3 & $1.68 \pm 0.01^{\mathrm{g}}$ & $3.01 \pm 0.01^{\mathrm{ed}}$ & $3.31 \pm 0.01^{\mathrm{g}}$ & $2.85 \pm 0.01^{\mathrm{f}}$ \\
\hline
\end{tabular}

$(A, b, c, \ldots)$ Means within the same letter in each column are not significantly different.

(T1)Pediococcus

(T2) pediococcus plus entrococcus

(T3) Bacillus licheniforis plus Bacillus Subtilis

\section{Performance index}

Results obtained that listed in Table 6 showed that treatments applied had no significant effect on the value of performance index at the first 2 weeks of age, however, highly significant variation due to treatments applied was observed when estimated at 2-4 and 4-6 weeks and during the whole experimental period 0-6 weeks.Supplementing diet with Bacillus licheniforis plus Bacillus Subtilis had the better performance index during the period from 0-2weeks (4.27\%), 2-4 weeks (16.79\%), 4-6 weeks $(32.07 \%)$ as well as during the whole experimental period $0-6$ weeks $(66.99 \%)$ followed by those fed diet with pediococcus plus entrococcus 4.00, 16.40, 31.26 and $64.72 \%$, respectively and then by those fed pediococcus alon 3.93, 16.08, 29.58and 61.22\%, respectively( Table 6).On the other hand, level of dietary supplementation showed highly significant effect $(p<0.001)$ on performance index during the period from (2-4), (4-6) and (0-6) weeks of age. In general all levels of feed additives had the superiority of the performance index values compared to controls (Table 6). Performance index increased in its magnitude as the level of dietary supplements increased reaching its maximum effect when birds fed diet supplemented with $2.0 \mathrm{~kg}$ probiotic /ton ration. Significant variation was detected due to the interaction effect between treatments applied and levels of dietary supplementation at all periods of estimation, except at the period from 0-2 weeks of age only. This effect differed within treatments applied. The higher values of performance index were found in birds fed diet supplemented with Bacillus licheniforis plus Bacillus Subtilis when interacted with either 1.5 or $2.0 \mathrm{~kg} / \mathrm{ton}$ ration. Obtained results agree with those obtained by ElGendi et al., (2000); Salim (2004); Namra et al., (2005); Awad et al., (2009) and Hegab, (2010) who concluded that synbiotic or probiotic displayed a greater efficacy as growth promoters for broilers.

\section{Economical efficiency}

From the results obtained in (Table 7), it could be concluded that, dietary probiotic supplementation applied showed highly significant effect on the value of economical efficiency. This may be attributed to the significant differences that were found in body weight gain and feed conversion. Supplementeing birds diet with Bacillus licheniforis plus Bacillus Subtilis had the highest values of economical efficiency (115.33\%) compared with those supplemented with pediococcus plus Entrococcus treatment $(112.50 \%)$ or pediococcus alone $(108.33 \%)$. Highly significant variation $(\mathrm{p}<0.001)$ was found in the economical efficiency due to the level of feed additives applied. Generally all levels of feed additives had the superiority of the economical efficiency values compared to control group. 
Table 6. Least squares means and standard error (LSM \pm S.E) for performance index of birds of different experimental groups as affected by dietary supplements applied.

\begin{tabular}{|c|c|c|c|c|c|}
\hline \multirow{2}{*}{\multicolumn{2}{|c|}{$\begin{array}{l}\text { Independent variables } \\
\text { and interaction }\end{array}$}} & \multicolumn{4}{|c|}{ Performance index $(\%)$ at } \\
\hline & & 0-2 Weeks & 2-4 Weeks & 4-6 Weeks & 0-6 Weeks \\
\hline \multirow{3}{*}{$\begin{array}{c}\text { treatments } \\
(\mathrm{T})\end{array}$} & (T1) & $3.93 \pm 0.15$ & $16.08 \pm 0.12^{b}$ & $29.58 \pm 0.12^{\mathrm{c}}$ & $61.22 \pm 0.19^{\mathrm{c}}$ \\
\hline & (T2) & $4.00 \pm 0.15$ & $16.40 \pm 0.12^{b}$ & $31.26 \pm 0.12^{b}$ & $64.72 \pm 0.19^{b}$ \\
\hline & (T3) & $4.27 \pm 0.15$ & $16.79 \pm 0.12^{\mathrm{a}}$ & $32.07 \pm 0.12^{\mathrm{a}}$ & $66.99 \pm 0.19^{\mathrm{a}}$ \\
\hline \multirow{4}{*}{$\begin{array}{l}\text { levels } \\
(\mathrm{L})\end{array}$} & L0( Control) & $3.78 \pm 0.18$ & $15.17 \pm 0.31^{\mathrm{c}}$ & $26.13 \pm 0.14^{\mathrm{c}}$ & $55.00 \pm 0.22^{\mathrm{c}}$ \\
\hline & L1(1.0kg/ton) & $4.04 \pm 0.18$ & $16.45 \pm 0.31^{b}$ & $31.51 \pm 0.14^{b}$ & $65.19 \pm 0.22^{b}$ \\
\hline & L2(1.5 kg/ton) & $4.27 \pm 0.18$ & $16.96 \pm 0.13^{\mathrm{a}}$ & $33.13 \pm 0.14^{\mathrm{a}}$ & $68.47 \pm 0.22^{\mathrm{a}}$ \\
\hline & L3( 2.0kg/ton) & $4.29 \pm 0.18$ & $17.11 \pm 0.31^{\mathrm{a}}$ & $33.15 \pm 0.14^{\mathrm{a}}$ & $68.59 \pm 0.22^{\mathrm{a}}$ \\
\hline \multirow{12}{*}{$\begin{array}{c}\text { Interaction } \\
(\mathbf{T} \times \mathbf{L})\end{array}$} & T1 $\times$ L0 & $3.78 \pm 0.31$ & $15.17 \pm 0.24^{\mathrm{c}}$ & $26.13 \pm 0.25^{\mathrm{f}}$ & $55.00 \pm 0.39^{\mathrm{h}}$ \\
\hline & T1× L1 & $3.79 \pm 0.31$ & $16.01 \pm 0.24^{\mathrm{a}}$ & $31.00 \pm 0.25^{\mathrm{e}}$ & $63.09 \pm 0.39^{g}$ \\
\hline & T1 $\times$ L2 & $4.07 \pm 0.31$ & $16.15 \pm 0.24^{b}$ & $30.26 \pm 0.25^{\mathrm{e}}$ & $63.17 \pm 0.39^{g}$ \\
\hline & T1× L3 & $4.10 \pm 0.31$ & $16.99 \pm 0.24^{\mathrm{a}}$ & $30.34 \pm 0.25^{\mathrm{e}}$ & $63.64 \pm 0.39^{\mathrm{a}}$ \\
\hline & $\mathrm{T} 2 \times \mathrm{LO}$ & $3.79 \pm 0.31$ & $15.17 \pm 0.24^{\mathrm{c}}$ & $26.13 \pm 0.25^{\mathrm{f}}$ & $55.00 \pm 0.39^{\mathrm{h}}$ \\
\hline & T2 $\times$ L1 & $3.97 \pm 0.31$ & $16.23 \pm 0.24^{b}$ & $31.75 \pm 0.25^{\mathrm{d}}$ & $65.34 \pm 0.39^{f}$ \\
\hline & $\mathrm{T} 2 \times \mathrm{L} 2$ & $4.10 \pm 0.31$ & $17.05 \pm 0.24^{\mathrm{a}}$ & $33.14 \pm 0.25^{\mathrm{c}}$ & $68.62 \pm 0.39^{d}$ \\
\hline & T2 2 L3 & $4.14 \pm 0.31$ & $17.15 \pm 0.24^{\mathrm{a}}$ & $34.01 \pm 0.25^{b}$ & $69.93 \pm 0.39^{c}$ \\
\hline & T3× L0 & $3.79 \pm 0.31$ & $15.17 \pm 0.24^{\mathrm{c}}$ & $26.13 \pm 0.25^{f}$ & $55.00 \pm 0.39^{h}$ \\
\hline & T3× L1 & $4.35 \pm 0.31$ & $17.13 \pm 0.24^{\mathrm{c}}$ & $31.79 \pm 0.25^{\mathrm{d}}$ & $67.15 \pm 0.39^{\mathrm{e}}$ \\
\hline & T3× L2 & $4.55 \pm 0.31$ & $17.68 \pm 0.24^{\mathrm{a}}$ & $35.40 \pm 0.25^{\mathrm{a}}$ & $73.62 \pm 0.39^{\mathrm{a}}$ \\
\hline & T3× L3 & $4.42 \pm 0.31$ & $17.19 \pm 0.24^{\mathrm{a}}$ & $34.98 \pm 0.25^{\mathrm{a}}$ & $72.20 \pm 0.39^{b}$ \\
\hline
\end{tabular}

$(\mathrm{A}, \mathrm{b}, \mathrm{c}, . .$.$) Means within the same letter in each column are not significantly different.$

(T1)Pediococcus

(T2) pediococcus plus entrococcus

(T3) Bacillus licheniforis plus Bacillus Subtilis

Table 7. Least squares means and standard error (LSM \pm S.E) for economical efficiency (\%) of birds of different experimental groups as affected by dietary supplements applied.
Independent variables

\section{Economical efficiency $(\%)$ at}

and interaction 6 weeks

\begin{tabular}{|c|c|c|}
\hline \multirow{3}{*}{$\begin{array}{c}\text { treatments } \\
(\mathrm{T})\end{array}$} & (T1) & $108.33 \pm 1.47^{b}$ \\
\hline & (T2) & $112.50 \pm 1.47^{\mathrm{ab}}$ \\
\hline & (T3) & $115.33 \pm 1.47^{\mathrm{a}}$ \\
\hline \multirow{4}{*}{$\begin{array}{l}\text { levels } \\
(\mathrm{L})\end{array}$} & L0( Control) & $100.00 \pm 1.70^{b}$ \\
\hline & L1(1.0kg/ton) & $113.11 \pm 1.70^{\mathrm{a}}$ \\
\hline & $\mathrm{L} 2(1.5 \mathrm{~kg} / \mathrm{ton})$ & $117.77 \pm 1.70^{\mathrm{a}}$ \\
\hline & L3( 2.0kg/ton) & $117.33 \pm 1.70^{\mathrm{a}}$ \\
\hline \multirow{12}{*}{$\begin{array}{l}\text { Interaction } \\
(\mathbf{T} \times \mathbf{L})\end{array}$} & $\mathrm{T} 1 \times \mathrm{L} 0$ & $100.00 \pm 1.70$ \\
\hline & $\mathrm{T} 1 \times \mathrm{L} 1$ & $111.00 \pm 2.94$ \\
\hline & $\mathrm{T} 1 \times \mathrm{L} 2$ & $111.33 \pm 2.94$ \\
\hline & $\mathrm{T} 1 \times \mathrm{L} 3$ & $111.00 \pm 2.94$ \\
\hline & $\mathrm{T} 2 \times \mathrm{L} 0$ & $100.00 \pm 1.70$ \\
\hline & $\mathrm{T} 2 \times \mathrm{L} 1$ & $112.00 \pm 2.94$ \\
\hline & $\mathrm{T} 2 \times \mathrm{L} 2$ & $118.66 \pm 2.94$ \\
\hline & $\mathrm{T} 2 \times \mathrm{L} 3$ & $119.33 \pm 2.94$ \\
\hline & $\mathrm{T} 3 \times \mathrm{L} 0$ & $100.00 \pm 1.70$ \\
\hline & $\mathrm{T} 3 \times \mathrm{L} 1$ & $116.33 \pm 2.94$ \\
\hline & $\mathrm{T} 3 \times \mathrm{L} 2$ & $123.33 \pm 2.94$ \\
\hline & $\mathrm{T} 3 \times \mathrm{L} 3$ & $121.66 \pm 2.94$ \\
\hline
\end{tabular}

$(\mathrm{A}, \mathrm{b}, \mathrm{c}, \ldots$.$) Means within the same letter in each column are not significantly different$

(T1)Pediococcus

(T2) pediococcus plus entrococcus

(T3) Bacillus licheniforis plus Bacillus Subtilis 
Economical efficiency value increased as the level of dietary supplementation increased reaching its maximum value at $1.5 \mathrm{~kg}$ probiotic/ton ration $(117.77 \%)$ then it decreased with increasing the level of dietary supplementation.On the other hand, no significant variation in the average of economical efficiency due to the interaction effect between treatments and level of dietary supplementations. The higher economical efficiency values were observed when Bacillus licheniforis plus Bacillus Subtilis applied at the level of either 2 or 3 (1.5 or $2 \mathrm{~kg} / \mathrm{ton}$ ration), respectively. This improvement could be attributed to improving the feed utilization or reducing the amount of feed required for unit of body weight gain.Obtained results agree with those found by Abdel-Azeem et al., (2001); Anjum et al., (2005); Ghazalah, et al, (2006); Abaza et al., (2008), Tollba and Mahmoud (2009) and Hegab (2010) who all reported that, probiotic improved economic efficiency in broilers and quail diets.

\section{Mortality rate percentage}

Inspection of data (Table 8 ) revealed that, highly significant variation $(\mathrm{p}<0.001)$ in mortality rate during the whole experimental period was found due to treatments applied.Chicks received pediococcus plus entrococcus strains recorded the lowest average of mortality rate $(6.24 \%)$ as compared with either Bacillus licheniforis plus Bacillus Subtilis (6.66\%) or pediococcus alone (7.91\%). Highly significant effect was observed in the average mortality rate due to the level of feed supplementation. All levels applied lowered average of mortality rate. The lowest average of mortality rate was observed in chicks received probiotics at a level of $1.5 \mathrm{~kg}$ probiotics per ton diet $(3.88 \%)$ followed by those fed $2 \mathrm{~kg} / \mathrm{ton}$ $(4.99 \%)$ then by $1 \mathrm{~kg} / \mathrm{ton}(5.55 \%)$.No significant effect on average mortality rate was found due to the interaction between treatments and level of dietary supplementations. The lowest average of mortality rate was observed when Bacillus licheniforis plus Bacillus Subtilis was applied at a level of either 1.5 or $2.0 \mathrm{~kg} /$ ton ration, respectively follwed by pediococcus plus entrococcus when applied with a level of $2 \mathrm{~kg} /$ ton ration, respectively. The significant decrease in mortality percentage due to treatment could be attributed to a reduction in colonization of enterpathogens in the gastrointestinal tract. Studies of Drake et al., (2003) and Higgins et al., (2007) demonstrated that administration of probiotic culture for 1 or 3 consecutive days was able to reduce Salmonella colonization in one day-old broiler chicks.

\section{Conclusion}

Bacillus licheniformis plus bacillus subtilis at alevel of 1.5 and $2.0 \mathrm{~kg}$ ton $\backslash$ ration seemed to achieve the favorable results and bing recommended from the economic point of view.

Table 8. Least squares means and standard error (LSM \pm S.E) for mortality rate $(\%)$ of birds of different experimental groups as affected by dietary supplements applied.

\begin{tabular}{|c|c|c|}
\hline \multicolumn{2}{|c|}{$\begin{array}{l}\text { Independent variables } \\
\text { and interaction }\end{array}$} & $\begin{array}{c}\text { Mortality rate }(\%) \text { at } \\
6 \text { weeks } \\
\end{array}$ \\
\hline \multirow{3}{*}{$\begin{array}{c}\text { treatments } \\
(\mathrm{T})\end{array}$} & (T1) & $7.91 \pm 0.29^{\mathrm{a}}$ \\
\hline & T2) & $6.24 \pm 0.29^{b}$ \\
\hline & (T3) & $6.66 \pm 0.29^{b}$ \\
\hline \multirow{4}{*}{$\begin{array}{l}\text { levels } \\
(\mathrm{L})\end{array}$} & LO( Control) & $13.33 \pm 0.33^{\mathrm{a}}$ \\
\hline & L1(1.0kg/ton) & $5.55 \pm 0.33^{b}$ \\
\hline & $\mathrm{L} 2(1.5 \mathrm{~kg} / \mathrm{ton})$ & $3.88 \pm 0.33^{\mathrm{c}}$ \\
\hline & $\mathrm{L} 3(2.0 \mathrm{~kg} / \mathrm{ton})$ & $4.99 \pm 0.33^{b}$ \\
\hline \multirow{12}{*}{$\begin{array}{l}\text { Interaction } \\
\quad(\mathbf{T} \times \mathbf{L})\end{array}$} & $\mathrm{T} 1 \times \mathrm{L} 0$ & $13.33 \pm 0.33$ \\
\hline & $\mathrm{T} 1 \times \mathrm{L} 1$ & $6.66 \pm 0.58$ \\
\hline & $\mathrm{T} 1 \times \mathrm{L} 2$ & $5.00 \pm 0.58$ \\
\hline & $\mathrm{T} 1 \times \mathrm{L} 3$ & $6.66 \pm 0.58$ \\
\hline & $\mathrm{T} 2 \times \mathrm{L} 0$ & $13.33 \pm 0.33$ \\
\hline & $\mathrm{T} 2 \times \mathrm{L} 1$ & $5.00 \pm 0.58$ \\
\hline & $\mathrm{T} 2 \times \mathrm{L} 2$ & $3.33 \pm 0.58$ \\
\hline & $\mathrm{T} 2 \times \mathrm{L} 3$ & $3.33 \pm 0.58$ \\
\hline & $\mathrm{T} 3 \times \mathrm{LO}$ & $13.33 \pm 0.33$ \\
\hline & $\mathrm{T} 3 \times \mathrm{L} 1$ & $5.00 \pm 0.58$ \\
\hline & $\mathrm{T} 3 \times \mathrm{L} 2$ & $3.33 \pm 0.58$ \\
\hline & T3× L3 & $5.00 \pm 0.58$ \\
\hline
\end{tabular}

(A, b, c) Means within the same letter in each column are not significantly different

(T1)Pediococcus

(T2) pediococcus plus entrococcus

(T3) Bacillus licheniforis plus Bacillus Subtilis 


\section{References}

Abaza, I.M., Shehata M.A., Shoieb M.S and Hassan I.I. (2008): Evaluation of some natural feed additive in growing chick diets. International Journal of Poult. Sci. 7, 9: 872-879.

Abdel-Azeem, F.; Ibrahim, A.; Faten, A. and Ali, G.M. Nematallah. (2001). Growth performance and some blood parameters of growing Japanese quail as influenced by dietary different protein levels and microbial probiotics supplementation. Egypt. Poult. Sci. 21(11): 465-489.

Al-Homidan, I; Alkhalf, A and Alhaj, M., (2010). Influence of probiotic supplementation on blood parameters and growth performance in broiler chickens. Saudi J. Biol. Sci. 17:219-225.

Anjum, M.I.; A.G. Khan; A. Azim and M. Afzal (2005). Effect of dietary supplementation of multi-strain prohiotic on broiler growth performance. Pakistan Vet. J., 25(1): 25-29.

Asmita K, Singh S. S; Neeruddin M. D and Singh K. C. P. (2001). Effect of probiotics on growth performance of meat type Japanese quail. Indian J. Poult. Sci., 36 (2): 233-234.

Awad, W.A.; Ghareeb, K.; Abdel-Raheern, S. and Bohrn, J. (2009). Effects of dietary inclusion of probiotic and synbiotic on growth, performance, organ weights, and intestinal histomorphology of broiler chickens. Poult. Sci. 88. 49-56.

Bailey, J.S., (1987). Factors affecting microbial competitive exclusion in poultry overviewoutstanding symposia in food science and technology. Food. Tech. July: 88-92.

Chafai, S.; Fatiha I. and Allow N. (2007): Bacterial prohiotic additive (Pecliococcus acidilactici) and its impact on broiler chickens health and performance. Animal health, animal welfare and biosecurity. The 13th International Congress in Animal Hygiene. Tartu. Estonia, 17-21 June 2007. Proc., 2. 820-825.

Chimote, M. J.; B. S. Barmase; A. S. Raut; A. P. Dhok and S. V. Kuralkar (2009). Effect of supplementation of probiotic and enzymes on performance of Japanese quails (2009). Veterinary World, Vol.2(6): 219-220.

Coates, M.E. and R. Fuller, (1977). The genotobiotic animal in the study of gut microbiology. In: R T.J. Clarke and T. Bauchop (Eds). Microbial ecology of the gut. Academic Press: London, pp: 311-346.

Drake, A.; Pixley, C.; Johnson, D.; Higgins, S. ;Nava, G.; Tellez, G.; Donoghue, D.; Donoghue, A. and Hargis, B.M. (2003). Evaluation of a simple in vitro selected probiotic consisting on nine non-pathogenic bacterial to prevent Salmonella infection in broiler chicks Poult. Sci. 82 (Suppl. 1): 32 (Abstr).

El-Gendi, G.M., Soliman, A.F. and Habib, A.G. (2000). Evaluating four feed additives for improving productive and metabolic performance of broiler chicks. Egypt. Poult. Sci., 20: 103-122.

Franco, L.D, Fondevila M; Lobera M.B and Castrillo. C (2005): Effect of combinations of organic acids in weaned pig diets on microbial species of digestive tract contents and their response on digestibility. Journal of Animal Physiology and Animal Nutrition 89, 88-93.

Fuller, R. (1989): Probiotics in man and animals. Journal of Applied bacteriology, 66, 365-378.

Ghazalah, A.A.; EI-Nagmy, K.Y., and Bahakim, A.S. (2006). Effect of using dry fat on performance, nutrients digestibility, carcass traits and blood constituents of broiler chicks. Egypt. Poult. Sci, 27 (II): (363-382).

Hegab, A.M.A. (2010). A trial to improve productivity and disease resistance in commercial broilers. Ph.D. Thesis, Faculty of Agriculture, Moshtohor, Benha University. Egypt.

Hideya, $H$ and Taku .S (2004). Effects of probiotic Bacillus cefeus toyoi on abdominal fat accumulation in the Japanese quail (Coturnix coturnix japonica). Animal Science Journa. volume 75, Issue 1, pages 37-41.

Higgins, J.P; Higgins, S.E; Vicente, J.L; Volfenden, A.D., Tellez, G. and Hargis, B. M. (2007). Temporal effects of lactic acid bacteria probiotic culture on salmonella in neonatal broilers. Poult. Sci .86: 1662-1666.

Jin, L. Z.; Y. W. Ho; M. A. AH, N; Abdullah, K. B. Ong, and S. Jalaludin. (1996). Adhesion of Lactobacillus isolates to intestinal epithelial cells of chicken. Lett. Appl. Microbiol. 22:229-232.

Jin, L.Z.; Ho, Y.W.; Abdullah, N. and Jalaludin, S. (2000). Digestive and bacterial enzyme activities in broilers fed diets supplemented with Lactobacillus Cultures. Poult. Sci. 79. 886-891.

Kamiya, T.; M. Shikano, T; Wada, M; Sasaki and T. Joh, (2008). The efficacy of probiotics in gastrointestinal disease. Nippon. Rinsho., 66: 1385-90.

Mountzouris, K. C.; P. Tsistsikos, E.; Kalamaru, S.; Nitsh, C.; Schatzmayr, and K. Fegeros. (2007). Evaluation of the efficacy of a probiotic containing Lactobacillus, Bifidobacierium, Enlerococcm, and Pediococcits strains in promoting broiler performance and modualting cecal microflora composition and metabolic activities. Poult. Sci. 86:309-317.

Nahashon, S.N.; U.S. Nakaue and L.W. Mirosh, (1992). Effect of direct-fed microbials on nutrient retention and production parameters of laying pullets. Poult Sci. 71(suppl. 1): 111 (Abstr).

Nahashon, S.N., U.S. Nakaue and L.W. Mirosh, (1993). Effect of direct fed microbials on nutrient retention and productive parameters of Single Comb White Leghorn pullets. Poult. Sci.. 72 (Suppl. 1): 87 (Abstr.). 
Namra, M. M. M.; Osman, A. M. R., and Hala M. Abdel Wahed (2005). Effect of replacing soybean seal with graded levels of dried yeast on broiler chick's performance. Egypt. Poult. Sci. (25) (IV): (987-1001).

North, M.O. (1981). Commercial chicken production manual. Avi publishing company. INC, West port. Connecticut, USA.

NRC, (1994). Nutrient Requirements of Poultry. 9th rev. ed. Nat). Acad. Press, Washington, DC. Whey permeate containing $80 \%$ lactose was obtained from Berner Foods Inc (Roscoe, 1L).

Pelicano, E. R. L.; Souza, P. A.; Souza, H. B. A.; Oba, A.;Boiago, M. M.; Zeola, N. M. B. L.; Scatolini, A. M.;Bertanha, V. A. and Lima, T. M. A., 9(2005): Carcass and cut yields and meat qualitative traits of broilers fed diets containing probiotics and prebiotics. Brazilian Journal of Poultry Science 7, 169-175.

Saarela, M.; Mogensen, G.; Fonden, R.; Matto, J. and Mattila Sandholm, T. (2000). Probiotic bacteria: safety, functional and technological properties. J. Biotechnol., 84: 197-215.

Salim, I.H.S. (2004). Effect of dietary protein and some feed additives on broiler performance. M.Sc. Thesis. Department of Animal Production, Fac. Agric. Cairo Univ., Giza, Egypt.

Sanders, M. E. and J. H. Veld. (1999). Bringing a probiotic containing functional food to the market: microbiological, product, regulatory and labeling issues. Antonie van Leeuwenhoek 76: 93-315.
Santos, G.D.L; J.R. O. B. Storch and C. Gilturnes (2005). Bacillus cereus var. toyoii and Saccharomyces boulardii increased feed efficiency in broilers infected with Salmonella enteritidis. Br.]Poult. Sci. 46. (4): 494-497.

SAS Institute Inc., (1996). SAS User's Guide Version 6. 12. SAS Institute Inc.

Silva, E.N., A.S. Teixeira, A.G. Bertechini, C.L. Ferreira and B.C. Ventura, (2000). Cienciae Agrotecnologia 24: Ed. Especial, 224-232.

Tarun. M, (2008). Growth performance of broilers fed with different strains of probiotics $16^{\text {th }} \mathrm{I}$ FOAM Organic World Congress, Modena, Italy, June 16-20, 2008 Archived at http://orgprints.org/11625.

Tollba, A. A. H. and R. M. Mahmoud (2009). How to control the broiler pathogenic intestinal flora under normal or heat stress conditions Animal Prod. Instit. Agric. Res. Center. Ministry of Agric. Giza, Egypt.

Willis, W.L.; Isikhuemhen, O.S., and Ibrahim S. A. (2007) Performance Assessment of Broiler Chickens Given Mushroom Extract Alone or in Combination with Probiotics. Poultry Science 86:1856-1860.

Yanbo, V. and Qing, G. (2010). Effect of probiotic on growth performance and digestive enzyme activity of Arbor Acres broilers. Research in Veterinary Science Volume 89, Issue 2. October 2010. Pages 163-167.

$$
\begin{aligned}
& \text { "تأثير اضافة البروبيوتيك على الاداء الانتاجى لكتاكيت السمان" } \\
& \text { محمد صفوت جادو و جعفر محمود الجندى و ثناء فؤاد محمدى واسامه حسن الجارحى و محمد السيد الخالع }
\end{aligned}
$$

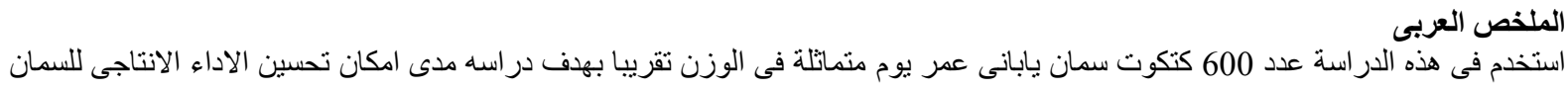

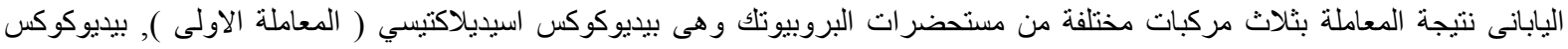

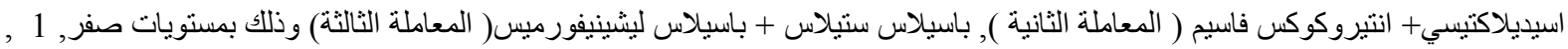

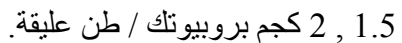

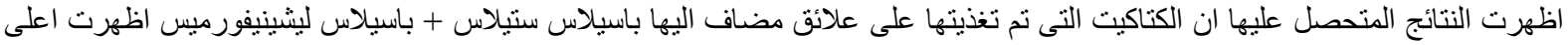

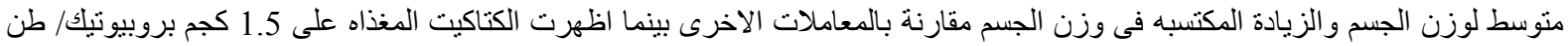

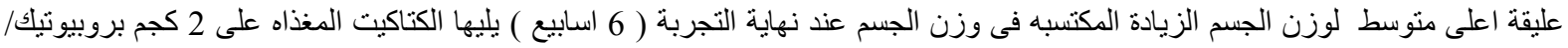

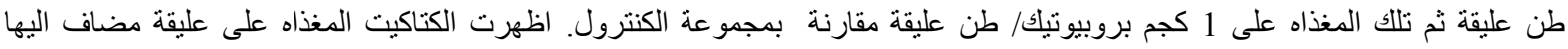

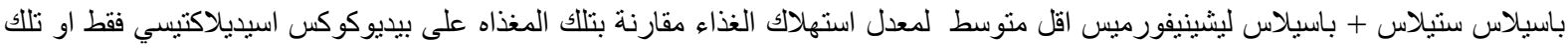

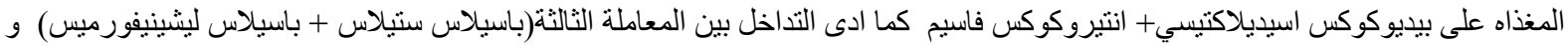

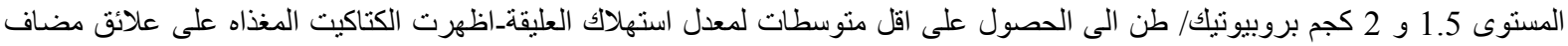

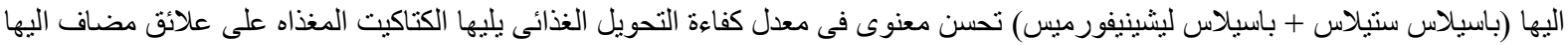

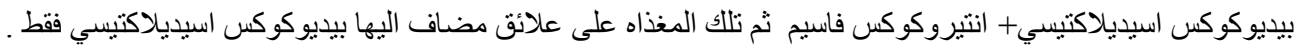

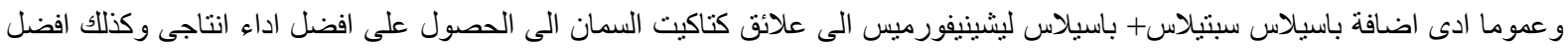

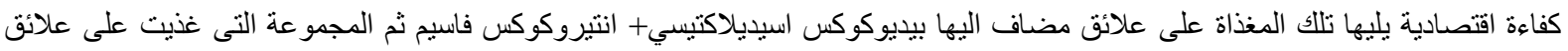
مضاف اليها بيديوكوكس اسيديلاكتيسي ومن جها اخرى لوحظت الفئ اقل معدلات لنسبة النفوق فى المجاميع التى تم اضافة البروبيوتلك الى علائقها

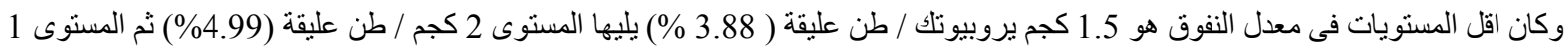

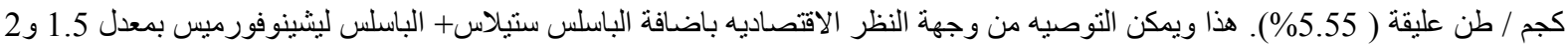

